\title{
Evaluation of the Effect of Vitamin D Supplementation on Anthropometric Indicators and Dietary Intake of Patients with Type 2 Diabetes
}

\author{
Mahnaz Zarei ${ }^{1}$, Mohammad Hassan Javanbakht ${ }^{2}$, \\ Hanieh Jafary ${ }^{3}$, Mahmoud Djalali*4
}

\begin{abstract}
Background: Various studies have shown that diabetes and its complications are associated with vitamin D deficiency. Due to the possible role of vitamin D in reducing the complications of diabetes and the high prevalence of its deficiency in Iran, this study was designed to investigate the effect of vitamin D supplementation on anthropometric indices and dietary intake of patients with type 2 diabetes.

Methods: This randomized clinical trial (RCT) study was performed on 74 patients with type 2 diabetes (T2DM). Patients randomly divided into two groups to receive vitamin D (VD) supplementation (100 $\mu \mathrm{g}$ or 4000 IU/day) or placebo for three months, randomization was based on the permutated-block method. Anthropometric indices including body weight (BW), body mass index (BMI) and waist circumference (WC) and physical activity, dietary intake were assessed by validated methods at the beginning and end of the trial.

Results: VD supplementation had not any significant differences in anthropometric indices, dietary intake and physical activity between the two groups.

Conclusions: Finally, it can be concluded, receiving 100 micrograms/day of VD for three months had no favourable effects on patients with T2DM..
\end{abstract}

Keywords: Anthropometric indices, Diabetes Mellitus, Dietary intake, Vitamin D.

\section{Introduction}

Diabetes is a group of metabolic disorders caused by complex reactions that occur between genetic factors and environmental factors. In diabetic patients, blood sugar rises, and chronic hyperglycemia by activating various cellular pathways causes damage to various organs, especially the eyes, heart, and kidneys (1). Type 2 diabetes, which is characterized by a combination of abnormal insulin secretion and increased insulin resistance, is responsible for more than 90 percent of adult diabetes. Because the progression of this type of diabetes is gradual and hyperglycemia develops gently, some patients with this type of diabetes are unaware of their disease for years, and this lack of awareness causes these patients to be more exposed to the vascular complications of diabetes (2). The global prevalence of diabetes in 2015 was reported to be $8.8 \%$ and is estimated to reach $10.4 \%$ by 2040 . The prevalence of diabetes in the urban population is significantly higher than in the rural population, and this finding indicates the important role of lifestyle in the occurrence of this chronic disease. Diabetes and its side effects impose a lot of medical costs on countries so that between 5 and $12 \%$ of their total annual medical budget is spent on controlling diabetes. Currently, 
$75 \%$ of people with diabetes live in low-income and middle-income countries, and the highest growth rate is expected in the coming years. The World Health Organization considers diabetes to be the most common endocrine disease in the world, causing 5 million deaths worldwide each year (3). According to the International Diabetes Federation (IDF), by 2030, Iran will become one of the most populous regions in the world in the field of diabetes (4). Also, the prevalence of diabetes in Iran is significantly higher than in neighboring countries such as Turkey and Pakistan, in other words, the prevalence of diabetes in Iran after Arab countries (5). Designing a proper diet for each person with the participation of the individual, which includes healthy food choices and controlling the number of calories received, is one of the useful nutritional interventions in these patients. Recommendations such as controlling total carbohydrate intake (carbohydrate counting), consuming whole grains and fiber, receiving omega-3 and monounsaturated fatty- acids and limiting food intake with a high glycemic index, limiting the intake of saturated and trans-fatty acids, can lead to the development of diabetes and prevent its side effects (6-8). Vitamin D was initially thought to play a role only in bone health, and its deficiency leads to bone abnormalities such as rickets in children, osteomalacia, and osteoporosis in adults (9). But today, other functions such as regulating immune function, regulating cell division and differentiation, inhibiting angiogenesis and proliferation of smooth muscle cells in the vascular wall, inhibiting beta growth factor in blood vessels (inhibiting vascular fibrosis) and regulating the renin-angiotensin system are considered for this vitamin $(10,11,12)$. Vitamin D deficiency has also been linked to many diseases, such as diabetes, depression, autoimmune diseases, cardiovascular disease, and cancer (9). Vitamin D, both through food and light in the blood, is transported by the proteinbound to vitamin $\mathrm{D}$ (and to a lesser extent by albumin about 10 to $15 \%$ ) and goes to the liver. So far, a number of studies have examined the link between vitamin $\mathrm{D}$ and diabetes, and it has been shown that there is a direct relationship between diabetes and vitamin $\mathrm{D}$ deficiency.
Vitamin D plays an important role in regulating insulin secretion (which is a calcium-dependent process) and glucose homeostasis. Vitamin D deficiency reduces glucose transporters. In addition, vitamin D deficiency is associated with increased fat mass and obesity, which is an important factor in insulin resistance and type 2 diabetes. Vitamin $\mathrm{D}$ is also involved in regulating the immune system (13).

In 2012, in a large population study in Australia Ganyon et al. found a significant association between vitamin $\mathrm{D}$ deficiency and a 5-year risk of metabolic syndrome and diabetes. In statistical analyzes, the effects of disruptive factors such as age, sex, race, season, latitude, smoking, family history of diabetes, physical activity, education, body mass index were eliminated. The researchers concluded that in Australian adults, low serum levels of vitamin D were associated with a fiveyear risk of developing metabolic syndrome and increased waist circumference, triglycerides, fasting sugar, and insulin resistance (14). Two reviews review and meta-analyses of the link between vitamin $\mathrm{D}$ and diabetes were conducted and published last year. In 2013, Green ova et al. found that lower levels of vitamin D were associated with obesity, high blood sugar, and decreased insulin sensitivity. Therefore, the authors concluded that vitamin D deficiency is a risk factor for obesity and insulin resistance (15).

\section{Materials and Methods \\ Compliance with ethical standards}

The present study was approved by the Ethics Committee of biology, Science and Research Branch, Islamic Azad University Tehran, Iran. The research was recorded on the Iranian Web site for registration of clinical trials (http: //www.irct.ir IRCT20170813035665N2).

In this study, 74 patients were enrolled to trial and randomly divided to two groups (37 in the intervention group and 37 were in the placebo group).

\section{Demographic information}

the gender, age, job status, education status, marital status, and drug history such as dose of metformin colloidal mud and aspirin) were collected at the beginning of intervention. 


\section{Anthropometric indices}

Bodyweight (BW), body mass index (BMI) and waist circumference and height of participants were measured by validated methods. The height was measured in a standing position without shoes, heels attached to the wall and a straight head using a tape measure with an accuracy of $0.5 \mathrm{~cm}$. Each patient weighed lightly without shoes and used the digital scale Seca with an accuracy of $100 \mathrm{~g}$ in the morning and on an empty stomach. The waist circumference was measured in the middle of the last rib and the lower extremity bone with minimal clothing by the non-elastic band meter. Body mass index was calculated based on the standard formula.

\section{Dietary assessment}

Dietary intakes were assessed using a 24-hour questionnaire at the beginning and end of the trial. Patients were asked to state their dietary status within 3 days ( 2 consecutive days and one day off). The values were then converted to hot using a home scale guide and finally analyzed by NUT4 software.

\section{Physical activity assessment}

The International Physical Activity Questionnaire (IPAQ) was used to assess the level of physical activity among participants. This questionnaire was completed at the beginning and end of the intervention. Using this questionnaire, which includes 7 short questions about the duration and number of days of the week that deal with moderate or heavy activity as well as walking and sitting time, collect the amount of physical activity performed other than daily activities during the past 1 week. The amount of physical activity was calculated as quantitative data by considering the coefficients related to the activity and recorded as MET. MET coefficients were calculated for walking 3.3 , average activity 4 , and heavy activity 8 , and these coefficients were multiplied by the minute and the number of days performed during the activity week, and its total as physical activity in the week was set.

\section{Statistical methods}

All of the analysis was performed with 25 SPSS statistical software and mean, and standard deviation were used to describe the data. Using the Kolmogorov-Smirnov test, the distribution of data was normalized. If the data is not normal, the variable conversion method was used to normalize the data. Medium (standard deviation) was used to describe quantitative data and frequency (percentage) was used for qualitative variables. An independent t-test was also used to compare the mean quantitative outcomes between the two groups. The Kai Square test was also used to compare qualitative factors between the two groups. ANCOVA statistical test was used to remove the effect of disruptive variables. The variable value of metformin, which was significantly different between the two groups at the beginning of the study, was considered as the distorter variable. Also, for comparison, a significance level of $p<0.05$ was considered for all analyzes.

\section{Results}

As can be seen in Table 1, the average age in the vitamin $\mathrm{D}$ group is 51.774 .7 and the average age in the placebo group is 52.668 5.68. Based on the independent $\mathrm{t}$-test, this difference between the two groups was not significant ( $\mathrm{p}=0.4)$. Based on this, it can be said that the age variable was similar between the two groups and is not considered as a distorting variable. About $17 \%$ of the education level in the vitamin D group and $8 \%$ of the patients in the placebo group are undergraduate education, $56 \%$ of the patients in the vitamin D group and $52 \%$ in the placebo group have a diploma and postgraduate education, $26 \%$ in the vitamin D group and $39 \%$ in the bachelor's degree group. And they were higher. According to the statistical test of Kai Square, this difference was not significant $(p=0.5)$. Based on this, it can be concluded that the level of education variable was similar between the two groups and was not considered as a disruptive factor. In terms of job status, $60 \%$ of patients were in the vitamin D group and $65 \%$ were employed in the placebo group, and $39 \%$ of those receiving vitamin D supplementation and $34 \%$ of those taking the placebo were unemployed. According to the statistical test of Kai Square, this difference was not significant $(p=0.7)$. Therefore, it can be concluded that the job situation between the two 
groups is similar and is not considered a disruptive factor. The duration of diabetes (since the diagnosis) was based on an independent t-test between two similar groups and did not differ significantly $(\mathrm{p}=0.3)$ and was not considered as a disruptive agent. Of the patients' medications, only the amount of metformin used between the two groups was significantly different $(\mathrm{p}=0.01)$ and was considered as a distorting variable. In the vitamin $\mathrm{D}$ receptor group, $52 \%$ of the patients and in the placebo group, $47 \%$ were female patients, which was not significant based on the chi-square statistical test $(p=0.7)$. Physical activity was recorded at the beginning and end of the study using the IPAQ questionnaire and became the metabolic equivalent (MET). As shown in the table before and after the intervention, there were no statistical differences in physical activity between the two groups $(\mathrm{p}<0.05)$ and participants did not significantly change their level of physical activity during the study $(\mathrm{p}<$ 0.05 ) Table 2.

Table 1. Baseline characteristics of patients in VD and placebo groups.

\begin{tabular}{|c|c|c|c|c|}
\hline \multicolumn{2}{|l|}{ Variable } & \multirow{2}{*}{$\begin{array}{l}\begin{array}{l}\text { Placebo } \\
(\mathbf{N}=37)\end{array} \\
(2.52) 12\end{array}$} & \multirow{2}{*}{$\begin{array}{l}\begin{array}{l}\text { Vitamin } D \\
(\mathbf{N}=37)\end{array} \\
(8.47) 11\end{array}$} & \multirow{2}{*}{$\begin{array}{l}\mathbf{p} \\
\text { value }\end{array}$} \\
\hline \multirow{2}{*}{ Sex } & Female & & & \\
\hline & Male & $(0.747) 11$ & (2.52) 12 & \\
\hline \multicolumn{2}{|l|}{ Age (year) } & $7.4 \pm 51.30$ & $5.6 \pm 52.40$ & $0.4^{\mathrm{b}}$ \\
\hline \multicolumn{2}{|c|}{ Diabetes duration (year) } & $0.62 \pm 0.75$ & $0.92 \pm 0.76$ & $0.3^{\mathrm{b}}$ \\
\hline \multirow{2}{*}{ Job status } & $\begin{array}{l}\text { Employee, self-employment, } \\
\text { retired }\end{array}$ & $14(60)$ & $15(65)$ & \multirow{2}{*}{$0.8^{\mathrm{a}}$} \\
\hline & Homeless and unemployed & $9(39)$ & $9(34)$ & \\
\hline \multirow{3}{*}{$\begin{array}{l}\text { Education } \\
\text { status }\end{array}$} & Under diploma & $4(17)$ & $2(8)$ & \multirow{3}{*}{$0.50^{\mathrm{a}}$} \\
\hline & Diploma and Postgraduate & $13(56)$ & $12(52)$ & \\
\hline & Bachelor's degree and higher & $6(26)$ & $9(39)$ & \\
\hline \multirow{2}{*}{ Marital status } & Single & $3(13)$ & $3(13)$ & $0.90^{\mathrm{a}}$ \\
\hline & Married & $20(87)$ & $20(87)$ & \\
\hline \multirow{3}{*}{ Drug history } & Metformin (mg/day) & $2.274 \pm 1065$ & $4.253 \pm 1282$ & $0.10^{\mathrm{b}}$ \\
\hline & Clay (mg/day) & $8.36 \pm 2.70$ & $1.29 \pm 1.73$ & $0.7^{\mathrm{b}}$ \\
\hline & Aspirin & $10(7.47)$ & $11(9.52)$ & $0.7^{\mathrm{a}}$ \\
\hline
\end{tabular}

\footnotetext{
${ }^{a}$ Chi-square test.

${ }^{\mathrm{b}}$ Independent t-test.

The values of the qualitative variables are as number (percentage).

The values of the quantitative variables are on mean \pm standard deviation (SD).

Significant levels were considered less than $p$-value $<0.05$.
} 
Table 2. Physical activity of patients in VD and placebo groups.

\begin{tabular}{lllll}
\hline Variable & & Vitamin $\mathbf{D}(\mathbf{N}=\mathbf{3 7})$ & $\begin{array}{l}\text { Placebo } \\
(\mathbf{N}=37)\end{array}$ & p-value \\
\cline { 1 - 4 } Low & Before & $12(52.1)$ & $17(73)$ & \\
\hline Medium & Before & $10(43.7)$ & $6(26.1)$ & \\
\hline Severe & Before & $1(4)$ & $0(0)$ & 0.30 \\
\hline Low & After & $12(52)$ & $15(65)$ & 0 \\
\hline Medium & After & $11(47)$ & $8(34)$ &
\end{tabular}

Due to the small number in the intense physical activity group, the moderate and severe groups merged, and the merged p-value was considered.

The data are expressed as numbers and percentages.

Significant levels were considered less than $\mathrm{p}$-value $<0.05$.

P-value based on Chi-square

Patients were compared for daily intake of energy, carbohydrates, protein, and fat at the beginning and end of the study (using the 24-hour questionnaire). As can be seen in Table 3-4, the two groups were not significantly different in terms of mean energy, fat, protein, and carbohydrate intake at the beginning and end of the study $(\mathrm{p}<0.05)$. Also, in comparing the beginning and end of the groups, there was no significant difference in terms of average energy, carbohydrate, protein, fat and carbohydrate intake $(\mathrm{p}<0.05)$ Table 3 . and these findings show that participants in both groups as They were asked not to change their diet. In addition, the findings on micronutrient intake in the two groups were shown in Table 45 based on the 24-hour questionnaire. As can be seen in Table 4 and Table 5, the two groups did not have a statistically significant difference in the mean intake of micronutrients at the beginning and end of the study $(\mathrm{p}<0.05)$. Also, in comparing the beginning and the end of the groups, no statistically significant difference was observed in terms of the average intake of micronutrients $(\mathrm{p}<0.05)$ Table 4.

Table 3. Macronutrient intake of patients in VD and placebo groups.

\begin{tabular}{|c|c|c|c|c|}
\hline Variables & & $\begin{array}{l}\text { Vitamin D } \\
(\mathrm{N}=37)\end{array}$ & $\begin{array}{l}\text { Placebo } \\
(\mathrm{N}=37)\end{array}$ & p-value ${ }^{b}$ \\
\hline \multirow{4}{*}{ Energy (kcal/day) } & Before & $1797.3 \pm 331.2$ & $1790.9 \pm 277.8$ & 0.7 \\
\hline & After & $1766.9 \pm 325.7$ & $1746.7 \pm 342.7$ & 0.6 \\
\hline & Changes & $-6.2 \pm 156.9$ & $-20.97 \pm 146.4$ & 0.7 \\
\hline & $\mathrm{P}$-value ${ }^{\mathrm{c}}$ & 0.8 & 0.5 & \\
\hline \multirow{4}{*}{$\begin{array}{l}\text { carbohydrate } \\
\text { (gr/day) }\end{array}$} & Before & $241.8 \pm 44.1$ & $255 \pm 53.6$ & 0.3 \\
\hline & After & $247.5 \pm 46.9$ & $250.7 \pm 49.3$ & 0.8 \\
\hline & Changes & $5.6 \pm 43.3$ & $-4.4 \pm 66.2$ & 0.5 \\
\hline & P-value ${ }^{c}$ & 0.5 & 0.7 & \\
\hline \multirow{4}{*}{ Protein (gr/day) } & Before & $55.8 \pm 12.4$ & $59.7 \pm 12$ & 0.2 \\
\hline & After & $58.7 \pm 11.3$ & $59 \pm 17.2$ & 0.7 \\
\hline & Changes & $2.80 \pm 12.2$ & $-70 \pm 17.2$ & 0.2 \\
\hline & P-value ${ }^{c}$ & 0.2 & 0.8 & \\
\hline \multirow{4}{*}{ Fat (gr/day) } & Before & $68.8 \pm 16.5$ & $71.5 \pm 17.3$ & 0.6 \\
\hline & After & $65.3 \pm 16.9$ & $68.9 \pm 16.5$ & 0.4 \\
\hline & Change & $3.2 \pm 28.7$ & $-2.7 \pm 19.7$ & 0.9 \\
\hline & P-value ${ }^{c}$ & 0.55 & 0.51 & \\
\hline
\end{tabular}

${ }^{\mathrm{b}} \mathrm{P}$ - value based on Independent t-test.

${ }^{\mathrm{c}} \mathrm{P}$-value based on Paired t- test.

Significant levels were considered less than $\mathrm{p}$-value $<0.05$. 
Table 4. Micronutrient intake of patients in VD and placebo groups.

\begin{tabular}{|c|c|c|c|c|}
\hline Variables & & $\begin{array}{l}\text { Vitamin D } \\
(\mathrm{N}=37)\end{array}$ & $\begin{array}{l}\text { Placebo } \\
(\mathbf{N}=37)\end{array}$ & p-value ${ }^{b}$ \\
\hline \multirow{4}{*}{ Vit C (gr) } & Before & $75.9 \pm 39.1$ & $96.5 \pm 44.5$ & 0.2 \\
\hline & After & $79.9 \pm 43.1$ & $84.67 \pm 39.6$ & 0.7 \\
\hline & Changes & $17.6 \pm 3.9$ & $-11.8 \pm 14.8$ & 0.2 \\
\hline & p-value & 0.7 & 0.1 & \\
\hline \multirow{4}{*}{ Vit E (gr) } & Before & $13.2 \pm 4.3$ & $11.4 \pm 3.4$ & 0.1 \\
\hline & After & $14.6 \pm 5.1$ & $13.5 \pm 7.1$ & 0.5 \\
\hline & Changes & $4.4 \pm 1.4$ & $6.1 \pm 2.07$ & 0.7 \\
\hline & $\mathrm{p}$-value ${ }^{\mathrm{c}}$ & 0.13 & 0.12 & \\
\hline \multirow{4}{*}{$\mathrm{Se}(\mathrm{mg})$} & Before & $0.059 \pm 0.038$ & $0.032 \pm 0.042$ & 0.1 \\
\hline & After & $0.029 \pm 0.05$ & $0.038 \pm 0.043$ & 0.5 \\
\hline & Changes & $0.005 \pm 0.009$ & $0.008 \pm 0.001$ & 0.3 \\
\hline & $\mathrm{p}$-value ${ }^{\mathrm{c}}$ & 0.1 & 0.9 & \\
\hline \multirow{4}{*}{ Vit D $(\mu \mathrm{g})$} & Before & $0.43 \pm 0.51$ & $0.42 \pm 0.53$ & 0.8 \\
\hline & After & $0.42 \pm 0.48$ & $0.27 \pm 0.49$ & 0.9 \\
\hline & Changes & $0.3 \pm-0.03$ & $0.28 \pm-0.04$ & 0.8 \\
\hline & p-value ${ }^{c}$ & 0.5 & 0.4 & \\
\hline \multirow{4}{*}{$\mathrm{Ca}(\mathrm{mg})$} & Before & $661 \pm 188$ & $602 \pm 117.1$ & 0.2 \\
\hline & After & $673 \pm 160$ & $607 \pm 124$ & 0.1 \\
\hline & Changes & $102.5 \pm 10.5$ & $98.03 \pm 4.10$ & 0.8 \\
\hline & p-value ${ }^{c}$ & 0.6 & 0.9 & \\
\hline
\end{tabular}

${ }^{\mathrm{b}}$ Independent T-Statistical Test.

${ }^{\mathrm{c}}$ Paired T-Statistical Test.

Significant levels were considered less than $\mathrm{p}$-value $<0.05$.

Table 5. Anthropometric indices of patients in VD and placebo groups.

\begin{tabular}{|c|c|c|c|c|}
\hline Variables & & $\begin{array}{l}\text { Vitamin D } \\
(\mathrm{N}=37)\end{array}$ & $\begin{array}{l}\text { Placebo } \\
(\mathrm{N}=37)\end{array}$ & p-value ${ }^{b}$ \\
\hline \multirow{4}{*}{ Weight (kg) } & Before & $9.8 \pm 73.7$ & $9.8 \pm 74.7$ & 0.7 \\
\hline & After & $9.7 \pm 73.2$ & $1.3 \pm 0.22$ & 0.6 \\
\hline & Change & $1.7 \pm 0.47$ & $1.3 \pm 0.22$ & 0.5 \\
\hline & p-value ${ }^{c}$ & 0.2 & 0.4 & \\
\hline \multirow{4}{*}{ BMI $\left(\mathrm{kg} / \mathrm{m}^{2}\right)$} & Before & $1.4 \pm 26.8$ & $1.6 \pm 27.5$ & 0.6 \\
\hline & After & $1.3 \pm 26.6$ & $1.7 \pm 27.3$ & 0.3 \\
\hline & Changes & $-0.06 \pm 0.5$ & $-0.06 \pm 0.5$ & 0.9 \\
\hline & P-value ${ }^{c}$ & 0.6 & 0.4 & \\
\hline \multirow{4}{*}{$\mathrm{WC}(\mathrm{cm})$} & Before & $7.4 \pm 96.6$ & $8.5 \pm 97.2$ & 0.8 \\
\hline & After & $7.5 \pm 96.5$ & $8.4 \pm 98.1$ & 0.8 \\
\hline & Change & $-0.1 \pm 0.7$ & $-0.2 \pm 1.03$ & 0.7 \\
\hline & p-value ${ }^{c}$ & 0.4 & 0.3 & \\
\hline
\end{tabular}

${ }_{\mathrm{b}} \mathrm{P}$-value based on Independent T-test.

${ }^{\mathrm{c}} \mathrm{P}$-value based on Paired t-test.

Significant levels were considered less than $\mathrm{p}$-value $<0.05$.

Table 5 shows the mean and standard deviation of the variables related to height, weight, and waist circumference and body mass index at the beginning of the study and at the end of the study in the two groups. At the beginning of the study, tonometry data did not show a significant difference between the two groups. In both groups, the mean body mass index before and after the study was in the overweight range. Comparison between the group and intragroup did not show a statistically significant statistical difference after 12 weeks of intervention $(\mathrm{p}<$ 
0.05). Comparison of the mean changes was also not significant for the findings of the survey among the studied groups $(\mathrm{p}<0.05)$.

\section{Discussion}

The results of this study showed the level of physical activity did not differ between the two groups studied before and after the intervention. Also, there were not significantly differences in terms of average energy intake, macronutrients and micronutrients. In interpreting this finding, it should be noted that due to the high number of entry criteria and non-entry into the study, the selected patients were similar in most variables and did not have statistically significant differences. According to these findings, the second hypothesis of the study is also confirmed. Therefore, the variables of physical activity level and average energy intake are not considered as disruptive factors of the study. Also, based on the findings of the present study, the mean weight, waist circumference and body mass index between the two groups were not statistically significant at the beginning of the study.

In its interpretation, it can be said that the similarity of the selected diabetic patients according to the various criteria for entering the study can be the reason for the lack of difference between the measurement indicators at the beginning of the study. In addition, based on our result, the mean weight changes, waist circumference and body mass index at the end of the study were not significantly different between the two groups, so the hypothesis is rejected.

\section{References}

1. Unwin N, Shaw J, Zimmet P, Alberti KG. Impaired glucose tolerance and impaired fasting glycaemia: the current status on definition and intervention. Diabet Med. 2002;19(9):708-23.

2. Franz MJ. Medical nutrition therapy for diabetes mellitus and hypoglycemia of nondiabetic origin. 2007.

3. Ogurtsova K, da Rocha Fernandes J, Huang Y, Linnenkamp U, Guariguata L, Cho NH, et al. IDF Diabetes Atlas: Global estimates for the prevalence of diabetes for 2015 and 2040. Diabetes Res Clin Pract. 2017;128:40-50.
Compared to the present study, in one clinical trial, a daily supplement of 1000 units of vitamin D did not help the participants' weight loss program (15). The results of another clinical trial study also showed that vitamin D supplementation weekly 20,000 units of vitamin A in people with a body mass index above 30 did not reduce waist circumference and the percentage of body fat mass in participants (16). In contrast to the findings of the present study, some studies had different results from our study. In one clinical trial, 100 patients with type 2 diabetes were randomly assigned to receive two doses of vitamin D-rich dairy $(1,000$ units) and dairy without fortification. After 12 weeks of intervention, weight, body mass index, and waist circumference decreased significantly in the vitamin D receptor group. The difference between the results of this study and the present study may be due to the high sample size and higher bioavailability of vitamin $\mathrm{D}$ intake through food and vitamin D intake with calcium (17).

Finally, it can be concluded that in this study, receiving 100 micrograms of vitamin $\mathrm{D}$ per day for 3 months had no effect on any of the anthropometric indices, level of physical activity and dietary intake of patients with type 2 diabetes in this study.

\section{Acknowledgements}

We greatly appreciate the assistance Tehran University of Medical Sciences and we also thank all volunteers for their participation in the study.

The authors declare that they have no conflicts of interests.

4. Cho N, Shaw J, Karuranga S, Huang Y, da Rocha Fernandes J, Ohlrogge A, et al. IDF Diabetes Atlas: Global estimates of diabetes prevalence for 2017 and projections for 2045. Diabetes Res clin Prac. 2018;138:271-281.

5. Esteghamati A, Etemad K, Koohpayehzadeh J, Abbasi M, Meysamie A, Noshad S, et al. Trends in the prevalence of diabetes and impaired fasting glucose in association with obesity in Iran: 20052011. Diabetes Res Clin Prac. 2014;103(2):319-27. 
6. Association AD. Standards of Medical Care in Diabetes-2017: summary of revision. Diabetes care. 2017;40 (Suppl 1):S4-S5.

7. Espositi K, Maiorino MI, Ceriello A, Giugliano D. Prevention and control of type 2 diabetes by Mediterranean diet: a systematic review. Diabetes research and clinical practice. 2010;89(2):97-102.

8. Hosseinzadeh P, Javanbakht MH, SA Mostafavi, Djalali M, Derakhshanian H, Hajianfar $\mathrm{H}$, et al. Brewer's Yeast Improves Glycemic Indices in Type 2 Diabetes Mellitus. Int J Prev Med. 2013; 4(10):1131-8.

9. Płudowski P, Karczmarewicz E, Bayer M, Carter G, Chlebna-Sokół D, Czech-Kowalska J, et al. Practical guidelines for the supplementation of vitamin D and the treatment of deficits in Central Europe - recommended vitamin D intakes in the general population and groups at risk of vitamin D deficiency. Endokrynol Pol. 2013;64(4):319-27.

10. Walsh JS, Bowles S, Evans AL. Vitamin D in obesity. Curr Opin Endocrinol Diabetes Obes. 2017;24(6):389-394.

11. Fleet JC, DeSmet M, Johnson R, Li Y. Vitamin

$\mathrm{D}$ and cancer: a review of molecular mechanisms.

Biochem J. 2012;441(1):61-76.

12. Nadimi H, Djazayery A, Javanbakht $\mathrm{MH}$, Dehpour A, Ghaedi E, Derakhshanian H, et al. The Effect of Vitamin D Supplementation on Serum and Muscle Irisin Levels, and FNDC5 Expression in Diabetic Rats. Reports of Biochemistry \& Molecular Biology. 2019;8(3):236-243.
13. Sung C-C, Liao M-T, Lu K-C, Wu C-C. Role of vitamin $\mathrm{D}$ in insulin resistance. J Biomed Biotechnol. 2012:634195.

14. Gagnon C, Lu ZX, Magliano DJ, Dunstan DW, Shaw JE, Zimmet PZ, et al. Low serum 25hydroxyvitamin $\mathrm{D}$ is associated with increased risk of the development of the metabolic syndrome at five years: results from a national, population-based prospective study (The Australian Diabetes, Obesity and Lifestyle Study: AusDiab). J Clin Endocrinol Metab. 2012;97(6):1953-61.

15. Grineva E, Karonova T, Micheeva E, Belyaeva $\mathrm{O}$, Nikitina I. Vitamin D deficiency is a risk factor for obesity and diabetes type 2 in women at late reproductive age. Aging (Albany NY). 2013;5(7):575-81.

16. Sneve M, Figenschau Y, Jorde R. Supplementation with cholecalciferol does not result in weight reduction in overweight and obese subjects. European journal of endocrinology. 2008;159(6):675-684.

17. Shab-Bidar S, Neyestani TR, Djazayery A, Eshraghian MR, Houshiarrad A, Gharavi A, et al. Regular consumption of vitamin D-fortified yogurt drink (Doogh) improved endothelial biomarkers in subjects with type 2 diabetes: a randomized doubleblind clinical trial. BMC Med. 2011;9:125. 